\title{
Dual Antithrombotic Therapy with Clopidogrel and Novel Oral Anticoagulants in Patients with Atrial Fibrillation Undergoing Percutaneous Coronary Intervention: A Real-world Study
}

Julia Kebernik (D) Martin Borlich · Ralph Tölg $\cdot$ Mohamed El-Mawardy •

Mohamed Abdel-Wahab · Gert Richardt

Received: January 29, 2018 / Published online: April 9, 2018

(C) The Author(s) 2018

\section{ABSTRACT}

Introduction: For patients with atrial fibrillation (AF) undergoing percutaneous coronary intervention (PCI), proper antithrombotic therapy is equivocal. Current guidelines recommend triple therapy, which carries a high risk of bleeding. Recent large trials suggest that dual therapy (DT) with novel oral anticoagulant (NOAC) plus $\mathrm{P}_{2} \mathrm{Y}_{12}$ inhibitor can be an appropriate alternative, but real-world data for this alternative are scarce and the optimal duration of DT has not yet been established.

Methods: This analysis was performed in a single-center prospective cohort. We investigated 216 PCI patients with indication for anticoagulation due to AF. After PCI patients received DT with reduced doses NOAC plus $\mathrm{P}_{2} \mathrm{Y}_{12}$ inhibitor for 6 months, which was followed by standard dose NOAC monotherapy. Efficacy endpoints were defined as cardiac death, myocardial infarction (MI), stent thrombosis (ST), and stroke. Safety endpoints

Enhanced digital features To view enhanced digital features for this article go to https://doi.org/10.6084/ m9.figshare.6035594.

J. Kebernik $(\varangle) \cdot$ M. Borlich · R. Tölg

M. El-Mawardy · M. Abdel-Wahab - G. Richardt

Heart Center Segeberger Kliniken, Bad Segeberg,

Germany

e-mail: jkebernik@gmx.de were bleeding events as defined by Bleeding Academic Consortium (BARC).

Results: Baseline characteristics of our study population were described by a $\mathrm{CHA}_{2} \mathrm{DS}_{2}$-VASc score of greater than 4 and a HAS-BLED score of greater than 3. After a mean follow-up of 18.7 months, efficacy events occurred in 12 patients $(5.6 \%)$. We observed three $(1.4 \%)$ cardiac deaths, two (0.9\%) MIs, six (2.8\%) strokes, and one $(0.5 \%)$ definite ST. After switching from DT to NOAC monotherapy after $6.3 \pm$ 1.7 months, there was no rebound of ischemic events. Bleeding events occurred in 34 patients (15.7\%) mainly under DT, while bleeding was less during NOAC monotherapy.

Conclusions: In this long-term study of highrisk and real-world AF-patients with PCI, DT with NOAC and $\mathrm{P}_{2} \mathrm{Y}_{12}$ inhibitor (6 months) followed by NOAC monotherapy was safe and effective.

Keywords: Anticoagulant agents; Antiplatelet agents; Atrial fibrillation; NOAC; Percutaneous coronary intervention

\section{INTRODUCTION}

Appropriate antithrombotic treatment in patients with atrial fibrillation (AF), who undergo OAC (PCI) is still unresolved. Dual antiplatelet therapy (DAPT) appears to be mandatory after PCI to reduce the risk of 
myocardial infarction (MI) and stent thrombosis (ST) [1], while long-term treatment with oral anticoagulation (OAC) is indicated in patients with $\mathrm{AF}$ to prevent stroke or systemic embolism [2]. Accordingly, current guidelines recommend triple therapy (TT) with DAPT plus OAC for at least 1 month after PCI [3, 4]. TT, however, substantially increases the risk of bleeding [5]. Favorable results were reported for a treatment strategy with dual therapy (DT) using single antiplatelet therapy plus vitamin $\mathrm{K}$ antagonists (VKAs) after PCI [7-10]. Novel oral anticoagulants (NOACs) are suitable alternatives to VKAs for stroke prevention in non-valvular $\mathrm{AF}$ and their use in clinical practice is increasing rapidly [11], also driven by guideline recommendation as first-line preference [12]. DT using NOACs and $\mathrm{P}_{2} \mathrm{Y}_{12}$ inhibitors may replace $\mathrm{TT}$ in $\mathrm{AF}$ patients with PCI, but so far, many questions are still open regarding this new antithrombotic strategy. First, it is unclear which medications are the best agents to combine. With several $\mathrm{P}^{2} \mathrm{Y}_{12}$ inhibitors and four NOAC agents, multiple potential drug combinations exist. Second, the optimal duration of DT and the dosage of NOAC is unclear. The recently published PIONEER AF-PCI trial [13] and REDUAL-PCI trial [14] as well as the ongoing ENTRUST-AF-PCI and the AUGUST studies aim to investigate the value of NOACs in combination with $\mathrm{P}_{2} \mathrm{Y}_{12}$ inhibitors. PIONEER AF and REDUAL-PCI showed that DT using NOAC significantly reduced bleeding compared to TT using VKA. Both trials, however, are underpowered for other clinical endpoints and thus clinical data in this area are of current interest. As the optimal antithrombotic treatment strategy for AF patients with PCI is still unresolved, the aim of this study was to assess safety and efficacy of 6 months DT with NOAC and $\mathrm{P}_{2} \mathrm{Y}_{12}$ inhibitor.

\section{METHODS}

\section{Study Design}

This retrospective analysis was performed in a single-center prospective cohort study aiming for consecutive enrolment of all AF patients with indication for PCI and OAC at the Heart
Center Segeberger Kliniken, Bad Segeberg, Germany. The current analysis includes 216 consecutive AF patients who underwent PCI from July 2013 to May 2016. Clinical follow-up was performed at least 6 months after the procedure. It was conducted by hospital visit or phone contact. A clinical event committee reviewed patient medical records including clinical events and hospital admissions in case of any adverse event.

\section{Interventional Procedure}

The indications for PCI and procedures were performed in accordance with the current guidelines and included stable coronary artery disease (CAD) as well as acute coronary syndromes (ACS). Procedural techniques were left to the operator's discretion and generally did not differ from non AF patients. To provide a real-world case study, all clinical presentations and procedural entities were included, as long as NOACs were not contraindicated. All patients received a loading dose of $500 \mathrm{mg}$ acetylsalicylic acid intravenously and $600 \mathrm{mg}$ clopidogrel or $60 \mathrm{mg}$ prasugrel orally. Unfractionated heparin was given intra-arterially during PCI at a dose of $5000-7000 \mathrm{U}$ with a target ACT time of $>250 \mathrm{~s}$. Patients did not take NOAC on the day of the procedure.

All procedures were performed transfemorally and arterial closure devices were used in all patients $\left(\mathrm{FemoSeal}^{\mathrm{TM}}\right.$, Terumo Europe $\mathrm{NV}$ ). In addition, access site was supplied with a pressure bandage for $3 \mathrm{~h}$ after removal of the sheath as in-house standard after transfemoral access.

\section{Endpoint Definition}

Pre-specified efficacy endpoints of this study were cardiac death, MI, ST, and stroke as defined by the Academic Research Consortium (ARC) [15]. Safety endpoint was the occurrence of bleeding events, defined by Bleeding Academic Consortium (BARC) [16]. 


\section{Antithrombotic Regimen}

All patients had indication for OAC due to nonvalvular AF with risk factors. After PCI, patients received reduced dosage NOAC plus a $\mathrm{P}_{2} \mathrm{Y}_{12}$ inhibitor for 6 months, followed by standard dosage of NOAC monotherapy. Most patients were already on NOAC at the time of the presentation and maintained the therapy, so the decision for a certain NOAC mainly depended on the referring physicians.

\section{Statistical Analysis}

All data were processed using Excel 2016 (Microsoft) and Prism 7.0 (GraphPad). The descriptive statistical characteristics for quantitative parameters are listed as mean \pm standard deviation for normally distributed data and as median with interquartile range for non-normally distributed data. Event-free survival for groups was constructed using Kaplan-Meier method.

\section{Compliance with Ethics Guidelines}

All procedures were in accordance with ethical standards of the responsible committee on human experimentation (institutional and national) and with the Helsinki Declaration of 1964, as revised in 2013. Informed written consent for the procedure was obtained from all patients.

\section{RESULTS}

A total of 220 patients with $\mathrm{AF}$ and need for OAC were consecutively treated with PCI from July 2013 until May 2016. Only four patients were lost to follow-up and excluded from final analysis. Detailed patients' baseline and clinical characteristics are summarized in Table 1 and 2. Mean age of the study population was $74.8 \pm 1.3$ years, and $80.1 \%$ were males. The comorbidity index was high, with $30.1 \%$ of the patients being diabetics, $13.4 \%$ with previous MI, $10.2 \%$ with previous ischemic events, and $22.7 \%$ with impaired renal function (glomerular
Table 1 Baseline characteristics

\begin{tabular}{ll}
\hline Age (years) mean \pm SD & $74.8 \pm 1.3$ \\
Male, $n(\%)$ & $174(80.1)$ \\
BMI $\left(\mathrm{kg} / \mathrm{m}^{2}\right)$, mean $\pm \mathrm{SD}$ & $28.4 \pm 4.95$ \\
Comorbidity and cardiac risk factors & \\
Diabetes mellitus, $n(\%)$ & $65(30.1)$ \\
Hypertension, $n(\%)$ & $201(93.1)$ \\
Dyslipidemia, $n(\%)$ & $112(51.8)$ \\
Current smoker, $n(\%)$ & $27(12.5)$ \\
Previous MI, $n$ (\%) & $29(13.4)$ \\
Previous CABG, $n$ (\%) & $24(11.1)$ \\
Previous cerebral ischemia, $n(\%)$ & $22(10.2)$ \\
Peripheral vessel disease, $n(\%)$ & $39(18.1)$ \\
Chronic renal failure (GFR <60), $n(\%)$ & $49(22.7)$ \\
Previous PCI, $n$ (\%) & $85(39.4)$ \\
\hline
\end{tabular}

Table 2 Clinical characteristics

\begin{tabular}{|c|c|}
\hline \multicolumn{2}{|l|}{ Clinical presentation } \\
\hline Stable angina, $n(\%)$ & $179(82.9)$ \\
\hline Acute coronary artery disease, $n(\%)$ & $37(17.1)$ \\
\hline Unstable angina, $n(\%)$ & $21(9.7)$ \\
\hline NSTEMI, $n(\%)$ & $10(4.6)$ \\
\hline STEMI, $n(\%)$ & $6(2.8)$ \\
\hline $\begin{array}{l}\text { Left ventricular ejection fraction, } \\
\text { mean } \pm S D\end{array}$ & $52.3 \pm 11.59$ \\
\hline $\begin{array}{l}\text { Left ventricular ejection fraction } \leq 30 \% \text {, } \\
n(\%)\end{array}$ & $19(8.8)$ \\
\hline $\mathrm{CHA}_{2} \mathrm{DS}_{2}$-Vasc score, mean $\pm S \mathrm{SD}$ & $4.3 \pm 1.24$ \\
\hline $\mathrm{CHA}_{2} \mathrm{DS}_{2}$-Vasc score, median (range) & $4(2-8)$ \\
\hline \multicolumn{2}{|l|}{ Coronary artery disease } \\
\hline $1 \mathrm{VD}, n(\%)$ & $58(26.8)$ \\
\hline $2 \mathrm{VD}, n(\%)$ & $74(34.3)$ \\
\hline $3 \mathrm{VD}, n(\%)$ & $84(38.9)$ \\
\hline
\end{tabular}


filtration rate $<60 \mathrm{ml} / \mathrm{min}$ ). The mean $\mathrm{CHA}_{2} \mathrm{DS}_{2}$-VASc score of our population was $4.4 \pm 1.2$ with a mean HAS-BLED score of $3.0 \pm 0.2$ representing a high-risk group for thromboembolic as well as bleeding events.

Three-quarters of the patients had multivessel coronary disease; 37 patients $(17.1 \%)$ presented with ACS. Stents were implanted in $93.5 \%$ in of the patients. On average, two stents were implanted with a mean total stent length of $35 \mathrm{~mm} ; 89.8 \%$ of the stents were new-generation drug-eluting stents (DES). A drug-eluting balloon PCI was performed in 5.5\% of the patients, $0.9 \%$ were treated with thrombus aspiration. Further procedural details are given in Table 3.

\section{Antithrombotic Regimen}

After the procedure, patients were treated with DT using reduced dosage NOAC, i.e., rivaroxaban $15 \mathrm{mg}$ once-daily in 182 patients (84.3\%),

Table 3 Procedural characteristics

\begin{tabular}{ll} 
Target vessel, $n(\%)$ & $17(6.2)$ \\
Left main coronary artery & $109(40.1)$ \\
Left anterior descending artery & $83(30.5)$ \\
Right coronary artery & $53(19.5)$ \\
Left circumflex artery & $10(3.7)$ \\
Bypass graft, $n(\%)$ & \\
Number of target vessels, $n(\%)$ & $168(77.8)$ \\
One target vessel & $40(18.5)$ \\
Two target vessels & $8(3.7)$ \\
Three target vessels & $2 \pm 1$ \\
Stents per patient, mean \pm SD & $35.25 \pm 25$ \\
Total stent length (mm), mean \pm SD & $194(89.8)$ \\
Drug-eluting stents, $n(\%)$ & $8(3.7)$ \\
Bare metal stents, $n(\%)$ & $12(5.5)$ \\
Drug-eluting balloon, $n(\%)$ & $2(0.9)$ \\
Other, $n$ (\%) & \\
\hline
\end{tabular}

dabigatran $110 \mathrm{mg}$ twice-daily in 17 patients (7.9\%), apixaban $2.5 \mathrm{mg}$ twice-daily in 16 patients $(7.4 \%)$, or edoxaban $30 \mathrm{mg}$ once-daily in one patient $(0.5 \%)$, starting the day after procedure in combination with either clopidogrel $(n=214 ; 99.1 \%)$ or prasugrel $(n=2 ; 0.9 \%)$. The duration of recommended DT was 6 months but was reduced to 4 weeks in two patients $(0.9 \%)$ and extended up to one year in 14 patients $(6.5 \%)$. The mean duration of recommended DT treatment was $6.3 \pm 1.7$ months (Fig. 1). DT was followed by a monotherapy of the respective NOAC using the regular dose as indicated for non-valvular AF.

\section{Outcomes}

Clinical follow-up was available in 216 patients (98.2\%) with a mean follow-up duration of 18.7 months. Bleeding was more frequently reported on DT than on NOAC monotherapy. During a mean DT of 6.3 months, bleeding events occurred in $11.5 \%$ of patients, while within the following NOAC monotherapy (mean, 11.9 months) only $3.2 \%$ of the patients experienced bleeding.

Bleeding events occurred in 34 of the patients (Table 4). Mild bleeding (BARC 1-2) was $6.5 \%$ on DT and $2.8 \%$ under NOAC monotherapy. Moderate and severe bleeding (BARC 3a-3c) was $4.6 \%$ on DT and $0.5 \%$ under NOAC monotherapy. There was only one fatal bleeding due to a hemorrhagic shock a few days

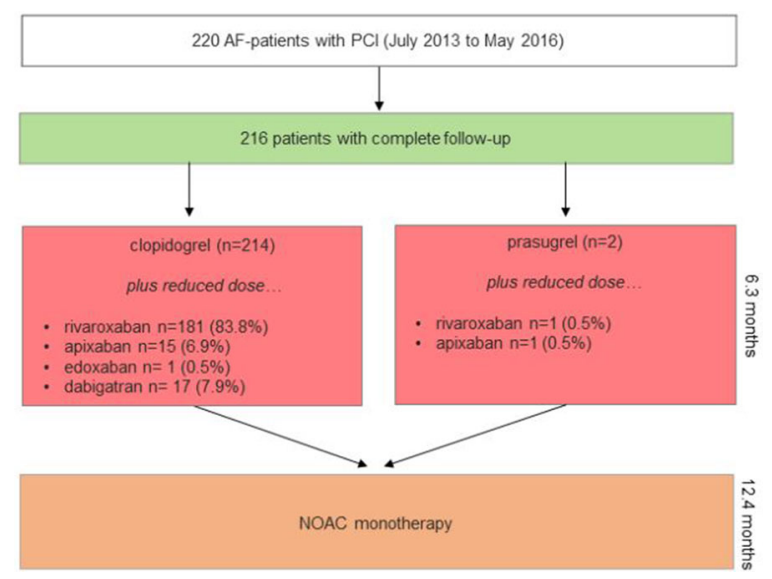

Fig. 1 Study design and patient enrolment 
Table 4 Bleeding events at follow-up

\begin{tabular}{ll}
\hline BARC type, $n$ (\%) & \\
BARC 1 & $13(6.0)$ \\
BARC 2 & $7(3.2)$ \\
BARC 3a & $4(1.8)$ \\
BARC 3b & $6(2.8)$ \\
BARC 3c & $3(1.4)$ \\
BARC 4 & 0 \\
BARC 5a & 0 \\
BARC 5b & $1(0.45)$ \\
TIMI type, $n$ (\%) & \\
Major & $7(3.2)$ \\
Minor & $5(3.2)$ \\
Minimal & $9(4.2)$ \\
Bleeding requiring medical attention & $13(6.0)$ \\
Clinically significant bleeding & $25(11.5)$ \\
\hline
\end{tabular}

after the index PCI while the patient was on DT, whereas no fatal bleeding happened during the following NOAC monotherapy. Two bleeding events (BARC 3a, 3c) occurred in patients who had changed recommended treatment and were on VKAs. The most common bleeding complications were recurrent epistaxis $(n=10)$ and access route related bleeding $(n=8)$. Further bleeding sites were gastrointestinal in six, intramuscular in two, trauma caused by collapse in two, intracerebral in one, and of unknown origin in four. Bleeding according to TIMI classification was detailed in Table 4 (major in seven $(3.2 \%)$, minor in five $(2.3 \%)$ and minimal in nine $(4.2 \%)$ ). Eighteen patients had changed the recommended treatment strategy mainly because of recurrent epistaxis, renal dysfunction, valve replacement, and left ventricular thrombus formation. Timing of all bleeding events is shown in Fig. 2.

Efficacy events occurred in 12 (5.6\%) of the patients (Table 5 ). All-cause mortality was $2.8 \%$ with a cardiac mortality of $1.4 \%$. Non-cardiovascular deaths were due to sepsis $(n=2)$, cancer $(n=2)$, paralytic ileus $(n=1)$, and renal failure $(n=1)$. We observed two MIs $(0.9 \%)$ and one definite ST $(0.5 \%)$. Six strokes were reported $(2.8 \%)$, all were ischemic. Only two of these occurred during DT period with reduced dose NOAC, whereas four patients suffered stroke during the following period of NOAC monotherapy in standard dose. Efficacy events occurred in $2.4 \%$ on DT (one cardiac death, one MI, one definite ST and two strokes), while 3.2\% were seen on following NOAC monotherapy (two cardiac deaths, one MI, and four strokes; Fig. 3). As shown in Fig. 3, ischemic events were equally distributed throughout the whole observation period.

\section{DISCUSSION}

The principal finding of this study is that 6-month DT consisting of NOAC plus $\mathrm{P}_{2} \mathrm{Y}_{12}$ inhibitor is safe and effective in high-risk $\mathrm{AF}$ patients with PCI. Moreover, with the de-escalation from DT to NOAC monotherapy, the risk of bleeding is further reduced.

For patients with indication for long-term $\mathrm{OAC}$ and PCI, guidelines recommend TT for at least 1 month [3, 4]. TT, however, increases the risk of fatal and non-fatal bleeding [5]. Despite recommendations for TT, real-world data reveal that discharge medication in most patients who had undergone PCI and require chronically anticoagulation consists of DAPT or DT using an OAC with single antiplatelet agent [6]. Several studies compared DT with TT. The WOEST trial randomized 573 patients with need for long-term OAC to DT (warfarin plus clopidogrel) or TT (warfarin plus clopidogrel plus acetylsalicylic acid in an open-label design. The group receiving DT had significantly lower rates of any bleeding and even less ischemic events within 1 year after PCI than the group receiving TT [7].

Despite the widespread use of NOACs, their role in $\mathrm{AF}$ patients undergoing $\mathrm{PCI}$ is widely unknown. It is unclear which dose of a NOAC should be used in combination with antiplatelet agents and which duration of DT is optimal. Currently it is also unknown whether antiplatelet therapy with NOAC is superior to 


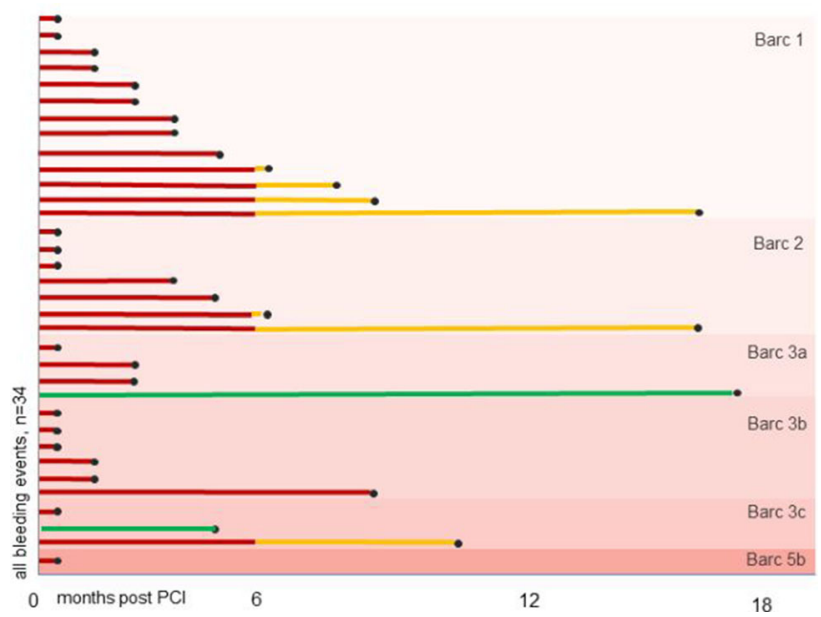

Fig. 2 Timing of all bleeding events. Bleeding events in patients with dual therapy (DT), NOAC monotherapy, and interruption of recommended antithrombotic treatment at some point before the occurrence of bleeding event

Table 5 Efficacy events at follow-up

\begin{tabular}{ll}
\hline Death, $n$ (\%) & $9(4.2)$ \\
Cardiac death & $3(1.4)$ \\
Vascular death & $0(0)$ \\
Non-cardiovascular death & $6(2.8)$ \\
Spontaneous MI, $n$ (\%) & $2(0.9)$ \\
Stent thrombosis, $n$ (\%) & \\
Definite & $1(0.5)$ \\
Probable & $0(0)$ \\
Possible & $0(0)$ \\
Stroke, $n$ (\%) & $6(2.8)$ \\
Ischemic & $6(2.8)$ \\
Hemorrhagic & $0(0)$ \\
\hline
\end{tabular}

antiplatelet therapy combined with VKA. Because addition of any type of single or dual antiplatelet therapy to VKA or NOAC anticoagulation significantly increases the risk of major bleeding, the European guidelines recommend maintaining the INR within 2-2.5 during triple or DT with VKA and reducing doses of NOAC [3]. To date, only two trials have randomly assigned patients requiring chronic anticoagulation and undergoing PCI to TT

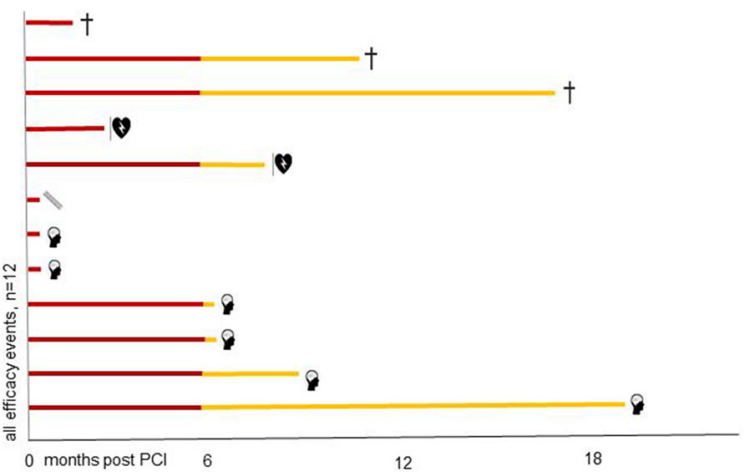

Fig. 3 Timing of all efficacy events. Efficacy events in patients with dual therapy (DT) and NOAC monotherapy at some point before the occurrence of efficacy event

versus DT with NOAC. Published in 2016, the PIONEER AF-PCI trial included 2214 AF patients who had just undergone PCI to compare the safety of two rivaroxaban treatment strategies (15 $\mathrm{mg}$ rivaroxaban plus a $\mathrm{P}_{2} \mathrm{Y}_{12}$ inhibitor or $2.5 \mathrm{mg}$ rivaroxaban twice daily plus DAPT) versus a TT using VKA. At 12 months, the primary outcome of clinically significant bleeding was lower in the rivaroxaban group than in the VKA group and lowest in the $15 \mathrm{mg}$ rivaroxaban group [13]. In the recently published REDUALPCI trial, 2752 patients were either treated with DT or with TT. The TT group received VKA plus a $\mathrm{P}_{2} \mathrm{Y}_{12}$ inhibitor and acetylsalicylic acid, while the DT group received dabigatran (110 mg or 
$150 \mathrm{mg}$ twice daily) plus a $\mathrm{P}_{2} \mathrm{Y}_{12}$ inhibitor [14]. These results reassured the findings of PIONEER-AF-PCI showing significantly lower rates of clinically relevant bleeding in the DT groups receiving reduced-dosage NOAC than in the group receiving TT. While these trials have been designed and powered for the primary safety endpoint of bleeding events, the secondary efficacy endpoint of cardiovascular events has not been adequately powered, even when enrolling several thousands of patients. This illuminates the difficulty in conducting a randomized controlled trial with its need for even larger cohorts to address this question. Adding more data supporting the efficacy of DT should therefore be of current interest and clinical importance.

We mainly utilized the DT regime of PIONEER-AF but shortened DT from 12 to 6 months. This was justified by a higher bleeding risk of DT than monotherapy and the declining risk of stent thrombosis within the first months after PCI. Furthermore, our patient cohort was mainly representative of stable CAD and high bleeding risk, so that 6 months of DT was an appropriate method.

With a mean age of nearly 75 years, a $\mathrm{CHA}_{2} \mathrm{DS}_{2}$-Vasc score of 4 , and a HAS-BLED score of 3 , our population has a higher patient-related risk for ischemic and bleeding events than patients in PIONEER-AF-PCI and in REDUALPCI. Moreover, the number and total length of stents indicate a high procedure-related risk. Nevertheless, the observed event rates are lower or at least similar to the two large RCTs. Clinically significant bleeding of $11.5 \%$ in our cohort is slightly lower than $16.8 \%$ in the respective DT arm in PIONEER-AF PCI (13) or 15.4\% in the DT arm with $110 \mathrm{mg}$ dabigatran in RE-DUAL PCI (14). Of note, the applied bleeding definitions differ slightly between both large trials, but may allow a cautious estimation. With respect to the efficacy endpoint events, we found a cardiovascular death rate of $1.4 \%$, which is lower than $2.4 \%$ in the respective DT arm of PIONEER-AF PCI and $5.6 \%$ in the DT arm with $110 \mathrm{mg}$ dabigatran in RE-DUAL PCI. The incidence of $0.9 \%$ myocardial infarction in our patients is lower than 3.0\% in PIONEER-A PCI and $4.5 \%$ in RE-DUAL PCI of the respective treatment arms.
The same applies with respect to definite and probable stent thromboses of $0.5 \%$ compared to $0.8 \%$ in PIONEER-AF PCI and $1.5 \%$ in RE-DUAL PCI. Of note, the triple therapy arms with double antiplatelet agent in the large randomized trials demonstrated a rate of stent thrombosis in the range of 0.7 to $0.9 \%$, which is not lower than the rate in our patients only receiving one antiplatelet agent. However, we found a somewhat higher rate of stroke with $2.8 \%$ compared to $1.3 \%$ in PIONEER-A PCI and $1.7 \%$ in RE-DUAL PCI of the respective treatment arms with DT. Of these six patients in our cohort with a stroke event, only two strokes $(0.9 \%)$ where noted during the phase of DT with a reduced NOAC dosage, whereas the majority of strokes where seen in a phase where NOAC was already administered in the approved dosage.

As we had a $98 \%$ follow-up rate and always full access to the patients' charts, it is very unlikely that our analysis suffers from serious underreporting of events. Our results rather support the contention that we should further reduce the aggressiveness of our antithrombotic therapy in elderly high-risk post PCI patients with AF. One might argue that 12 months of DT would have been more beneficial than 6 months of DT in our patients. DT, however, did not prevent the progression of thrombotic complications between month 6 and 12 in the RCTs, while our strategy halted bleeding in the same period after PCI.

\section{Study Limitations}

This study has all the limitations of a singlecenter observational study. The sample size of the study was small and no inference about comparative outcomes can be made due to lack of a control group. Secondly, different from the RCTs, our collective comprises mainly patients with stable CAD, which may compromise valid comparison to other published studies.

\section{CONCLUSIONS}

This single-center analysis suggests that 6 months of DT using a reduced dosage of 
NOAC plus $\mathrm{P}_{2} \mathrm{Y}_{12}$ inhibitor followed by standard dosage NOAC monotherapy is a safe and effective antithrombotic strategy for PCI patients with $\mathrm{AF}$ and need of OAC.

\section{ACKNOWLEDGEMENTS}

Funding. No funding or sponsorship was received for this study or publication of this article.

Authorship. All named authors meet the International Committee of Medical Journal Editors (ICMJE) criteria for authorship for this article, take responsibility for the integrity of the work as a whole, and have given final approval for the version to be published.

Thanking Participants. We thank the participants of the study.

Disclosures. Mohamed Abdel-Wahab reports receiving institutional research grants from St. Jude Medical and Biotronik and lecture fees from Edwards Lifesciences and Boston Scientific. Mohamed Abdel-Wahab is a proctor for Boston Scientific. Gert Richardt reports receiving institutional research grants from St. Jude Medical and Biotronik and lecture fees from Edwards Lifesciences and Boston Scientific. Ralph Tölg reports receiving lecture and advisory board fees from Abbott Vascular and Biotronik. Julia Kebernik, Martin Borlich and Mohamed ElMawardy have nothing to disclose.

Compliance with Ethics Guidelines. All procedures followed were in accordance with ethical standards of the responsible committee on human experimentation (institutional and national) and with the Helsinki Declaration of 1964, as revised in 2013. Informed written consent for the procedure was obtained from all patients.

Data Availability. The datasets during and/ or analyzed during the current study are available from the corresponding author on reasonable request.
Open Access. This article is distributed under the terms of the Creative Commons Attribution-NonCommercial 4.0 International License (http://creativecommons.org/licenses/ by-nc/4.0/), which permits any noncommercial use, distribution, and reproduction in any medium, provided you give appropriate credit to the original author(s) and the source, provide a link to the Creative Commons license, and indicate if changes were made.

\section{REFERENCES}

1. Levine GN, Bates ER, Blankenship JC, et al. 2015 ACC/AHA/SCAI Focused Update on Primary Percutaneous Coronary Intervention for Patients With ST-Elevation Myocardial Infarction: An Update of the 2011 ACCF/AHA/SCAI Guideline for Percutaneous Coronary Intervention and the 2013 ACCF/ AHA Guideline for the Management of ST-Elevation Myocardial Infarction: A Report of the American College of Cardiology/American Heart Association Task Force on Clinical Practice Guidelines and the Society for Cardiovascular Angiography and Interventions. Circulation. 2016;133:1135-47.

2. Kirchhof P, Benussi S, Kotecha D, et al. ESC Guidelines for the management of atrial fibrillation developed in collaboration with EACTS. Eur Heart J. 2015;37:2893-962.

3. Levine GN, Bates ER, Bittl JA, et al. 2016 ACC/AHA Guideline Focused Update on Duration of Dual Antiplatelet Therapy in Patients With Coronary Artery Disease: a Report of the American College of Cardiology/American Heart Association Task Force on Clinical Practice Guidelines: an Update of the 2011 ACCF/AHA/SCAI Guideline for Percutaneous Coronary Intervention, 2011 ACCF/AHA Guideline for Coronary Artery Bypass Graft Surgery, 2012 ACC/AHA/ACP/AATS/PCNA/SCAI/STS Guideline for the Diagnosis and Management of Patients With Stable Ischemic Heart Disease, 2013 ACCF/AHA Guideline for the Management of ST-Elevation Myocardial Infarction, 2014 AHA/ACC Guideline for the Management of Patients With Non-ST-Elevation Acute Coronary Syndromes, and 2014 ACC/ AHA Guideline on Perioperative Cardiovascular Evaluation and Management of Patients Undergoing Noncardiac Surgery. Circulation. 2016. https:// doi.org/10.1161/CIR.0000000000000404.

4. Valgimigli M, Bueno H, Byrne RA, et al. 2017 ESC focused update on dual antiplatelet therapy in coronary artery disease developed in collaboration with EACTS: the Task Force for dual antiplatelet 
therapy in coronary artery disease of the European Society of Cardiology (ESC) and of the European Association for Cardio-Thoracic Surgery (EACTS). Eur Heart J. 2017. https://doi.org/10.1093/ eurheartj/ehx419.

5. Hansen ML, Srensen R, Clausen MT, et al. Risk of bleeding with single, dual, or triple therapy with warfarin, aspirin, and clopidogrel in patients with atrial fibrillation. Arch Intern Med. 2010;170:1433-41.

6. Maier B, Hagenbarth C, Theres $H$, et al. Antithrombotic therapy in patients with atrial fibrillation and acute coronary syndrome in the real world: data from the Berlin AfibACS registry. Cardiol J. 2014;21:465-73.

7. Dewilde WJ, Oirbans T, Verheugt FW, WOEST study investigators, et al. Use of clopidogrel with or without aspirin in patients taking oral anticoagulant therapy and undergoing percutaneous coronary intervention: an open-label, randomised, controlled trial. Lancet. 2013;381:1107-15.

8. Ruiz-Nodar JM, Marín F, Hurtado JA, et al. Anticoagulant and antiplatelet therapy use in 426 patients with atrial fibrillation undergoing percutaneous coronary intervention and stent implantation implications for bleeding risk and prognosis. J Am Coll Cardiol. 2008;51:818-25.

9. Karjalainen PP, Porela P, Ylitalo A, et al. Safety and efficacy of combined antiplatelet-warfarin therapy after coronary stenting. Eur Heart J. 2007;28:726-32.
10. Denas G, Padayattil Jose S, Gresele P, et al. Major bleeding in patients undergoing PCI and triple or dual antithrombotic therapy: a parallel-cohort study. J Thromb Thrombolysis. 2013;35:178-84.

11. Olesen JB, Sorensen R, Hansen ML, et al. Non-vitamin $\mathrm{K}$ antagonist oral anticoagulation agents in anticoagulant naive atrial fibrillation patients: Danish nation-wide descriptive data 2011-2013. Europace. 2015;17:187-93.

12. Kirchhof P, Benussi S, Kotecha D, et al. 2016 ESC Guidelines for the management of atrial fibrillation developed in collaboration with EACTS. Eur J Cardiothorac Surg. 2016. https://doi.org/10.1093/ejcts/ ezw313.

13. Gibson CM, Mehran R, Bode C, et al. Prevention of bleeding in patients with atrial fibrillation undergoing PCI. N Engl J Med. 2016;375:2423-34.

14. Cannon CP, Bhatt DL, Oldgren J, RE-DUAL PCI Steering Committee and Investigators, et al. Dual antithrombotic therapy with dabigatran after PCI in atrial fibrillation. N Engl J Med. 2017;377:1513-24.

15. Cutlip DE, Windecker S, Mehran R, Academic Research Consortium, et al. Clinical end points in coronary stent trials: a case for standardized definitions. Circulation. 2007;115:2344-51.

16. Mehran R, Rao SV, Bhatt DL, et al. Standardized bleeding definitions for cardiovascular clinical trials: a consensus report from the Bleeding Academic Research Consortium. Circulation. 2011;123:2736-47. 